\title{
Supervised walking groups to increase physical activity in elderly women with and without hypertension: effect on pulse wave velocity
}

\author{
Francesco Fantin ${ }^{1}$, Andrea Rossi ${ }^{1}$, Susanna Morgante ${ }^{2}$, Diego Soave ${ }^{2}$, Luisa Bissoli ${ }^{1}$, Marco Cazzadori $^{1}$, \\ Maria Elena Vivian ${ }^{1}$, Massimo Valsecchi ${ }^{2}$ and Mauro Zamboni ${ }^{1}$
}

To date, only a few studies have evaluated the effect of physical activity on PWVcf in the elderly. In the current study, 21 community-dwelling women, with a mean age of $68.19 \pm 5.72$ years and a mean BMI of $28.63 \pm 4.69 \mathrm{~kg} \mathrm{~m}^{-2}$, participated in moderate physical activity sessions for $1 \mathrm{~h}$ per day and 2 days each week under the supervision of a qualified physical education instructor for a total of 24 weeks. At the beginning of the study, at 3 months and at 6 months, the study participants' body weight, waist circumference, sagittal abdominal diameter (SAD) and body composition by dual energy X-ray absorptiometry (DEXA) were recorded along with the participants' Physical Activity Scale for the Elderly questionnaire. Total low-density-lipoprotein and high-density lipoprotein cholesterol; triglycerides (TGs) and $\mathrm{HbA1c}$; blood pressure (BP); and arterial stiffness, as determined by carotid-femoral and carotid-radial pulse wave velocity (PWVcf, PWVcr), were also assessed. During the follow-up period, the waist and SAD significantly decreased, whereas fat-free mass, BMI and weight did not decrease. A significant decline in TGs was observed. A significant decline in PWVcf, even after adjusting for mean arterial pressure, heart rate triglycerides and waist diameter changes, was observed. In a sub-analysis that examined the effect of physical activity separately in the hypertensive and normotensive subjects, we observed a significant decline in PWVcf in the hypertensive subjects and a nonsignificant tendency in the normotensive subjects. The data showed an association between light aerobic physical activity in the elderly and decreased PWVcf, even after adjusting for changes in systolic BP (SBP), TGs and central adiposity. These results suggest a beneficial effect of moderate physical activity on subclinical vascular damage, particularly in hypertensive subjects.

Hypertension Research (2012) 35, 988-993; doi:10.1038/hr.2012.85; published online 5 July 2012

Keywords: cardiovascular risk factors; elderly; physical activity; pulse wave velocity

\section{INTRODUCTION}

Exercise may reduce cardiovascular (CV) morbidity and mortality ${ }^{1}$ and decrease several CV risk factors. The beneficial effect of aerobic physical activity on $\mathrm{CV}$ risk factors has been reported even in the elderly. ${ }^{2-6}$

Cross-sectional studies have shown that physical activity is associated with lower values of pulse wave velocity carotid-femoral (PWVcf), a surrogate marker of subclinical vascular damage. ${ }^{7-9}$

To date, only a few studies have evaluated the effect of physical activity on PWVcf in the elderly. Madden et al. ${ }^{10}$ showed that aerobic exercise reduced arterial stiffness (that is, PWVcf and pulse wave velocity carotid-radial (PWVcr)) in a group of 36 elderly diabetic patients, whereas non-aerobic exercise did not demonstrate an effect.

No studies have supported the efficacy of moderate physical activity in improving arterial stiffness in elderly subjects with hypertension.
Research has shown that the significant effect of physical activity on $\mathrm{CV}$ risk factors is limited to the length of the exercise session. Among older adults, the amount of time spent performing light physical activity has been associated with a lower PWVcf. ${ }^{11}$ Furthermore, a minimum threshold ( $>625 \mathrm{~kJ}$ per day) of physical activity has been linked to an improvement in health. ${ }^{12}$

However, the efficacy of physical activity in reducing CV risk factors is strongly limited by the low rate of compliance, ${ }^{12}$ and it is possible to hypothesize that higher levels of physical activity, in terms of intensity, duration and frequency per week, may lower compliance.

The aim of the present study was to assess the effect of a moderate (60-min exercise sessions of walking twice per week-approximately 7-8 METs per week), 6-month aerobic exercise program on CV risk factors and PWV in a group of apparently healthy elderly women with and without hypertension. 


\section{METHODS}

\section{Subjects}

Seventy-seven women were invited to an informative session about the study, and 55 women participated in the session. Of those who attended the session, 26 women refused to participate in the study. A total of 27 women completed the 3-month evaluation; of these women, six did not complete the 6-month evaluation (five dropped out and one was hospitalized).

A final sample of 21 women, with ages ranging from 60 to 80 years $\left(68.19 \pm 5.72\right.$ years) and BMI ranging from 22.74 to $41.2 \mathrm{~kg} \mathrm{~m}^{-2}$ $\left(28.63 \pm 4.69 \mathrm{~kg} \mathrm{~m}^{-2}\right)$, completed the 6-month follow-up of the study.

All subjects had stable weight during the 6 months before study, and none of the subjects presented with diabetes; cancer; liver, renal or thyroid disease; or $\mathrm{CV}$ diseases (except hypertension). All of the subjects were non-smokers, and none of the subjects received lipid-lowering therapy. All women were postmenopausal, but none received hormone-replacement therapy. The subjects underwent a careful clinical assessment before the beginning of the study. Forty-five percent $(10 / 21)$ of the study population was hypertensive. Of the patients undergoing antihypertensive treatment $(n=10)$, three patients used both ACE inhibitors and calcium-channel blockers and seven used only ACE inhibitors. None of these patients modified their therapy during the 6-month duration of the study.

The study was approved by the Ethical Committee of Verona Hospital.

All of the subjects were evaluated at the beginning of the study, at 3 months and at 6 months.

\section{Anthropometric variables}

With the subjects barefoot and wearing light indoor clothing, body weight was measured to the nearest $0.1 \mathrm{~kg}$ (Salus scale, Milan, Italy) and height to the nearest $0.5 \mathrm{~cm}$ using a stadiometer (Salus stadiometer). The BMI was calculated as body weight adjusted by stature $\left(\mathrm{kg} \mathrm{m}^{-2}\right)$. The waist circumference was obtained with a measuring tape at the level of the narrowest part of the torso as viewed anteriorly.

The sagittal abdominal diameter (SAD) was measured with a portable sliding beam abdominal caliper while the subjects were in the supine position. The SAD was measured as the largest supine anteroposterior diameter between the xiphoid process and the umbilicus. ${ }^{13}$ The subjects were asked to inhale and exhale gently, and the arm of the caliper was lowered to touch the abdominal wall without compression. All subjects were examined on a standard flat hospital bed.

\section{Blood pressure measurements}

While the subject was in the supine position, brachial blood pressure (BP) was measured thrice in a time frame of 15 min using a mercury sphygmomanometer on the subject's left arm. The mean of three readings was considered to be the subject's BP. The BP was recorded immediately before the tonometric recording. The subject's conditions were standardized according to established guidelines as follows: all subjects had fasted and refrained from the consumption of alcohol or caffeine for the preceding 24-h period. Mean arterial pressure (MAP) was calculated by adding 1/3 of the pulse pressure (systolic minus diastolic) to the diastolic pressure.

\section{Arterial stiffness measurements}

Arterial stiffness was measured using the Complior SP device (Artech Medical, Pantin, France), a semiautomated device that utilizes two pressure transducers. ${ }^{14}$ All measurements were performed after $30 \mathrm{~min}$ of supine rest, and the temperature was controlled $\left(25 \pm 1{ }^{\circ} \mathrm{C}\right)$.

Each pressure transducer measures the pulse-wave form at each site, allowing one to measure transit time of the pulse wave between the two locations.

The transducers were placed over the carotid and femoral or radial arteries to measure indices of central and peripheral arterial stiffness (that is, PWVcf and PWVcr, respectively). ${ }^{14}$ The time delay was measured using the point of maximal upstroke during systole.

PWV was calculated from these digitally recorded (sampling rate $4000 \mathrm{~Hz}$ ) transducer measures.

\section{Biochemical analyses}

Venous blood samples for all metabolic assessments were obtained after the subjects fasted overnight. Plasma glucose was measured with a glucose analyzer (Beckman Instruments Inc., Palo Alto, CA, USA). The intraassay CV was $1.5 \%$.

Cholesterol and triacylglycerol concentrations were determined with an automated enzymatic method (Autoanalyzer; Technicon, Tarrytown, NY, USA). High-density lipoprotein cholesterol was measured using the method of Warnick and Albers. Low-density-lipoprotein cholesterol was calculated using the Friedwald formula. HbAlc was measured by high-performance liquid chromatography (Bio-Rad Diagnostics Group, Hercules, CA, USA).

\section{Dual energy X-ray absorptiometry}

Total body fat, trunk and appendicular soft tissue, leg soft tissue and total fatfree mass were determined using the DEXA Hologic QDR 4500 (Hologic Inc., Bedford, MA, USA) fan beam densitometer with software version 8.21.

The characteristics and physical concepts of DEXA measurements have been described elsewhere. ${ }^{15}$ All metal objects (for example, jewelry, snaps and belts) were removed. The measurements were taken with the subject positioned supine on the scanning table. Radiation exposure was less than $8 \mathrm{mSv}$, and the mean measurement time was $6 \mathrm{~min}$. Daily quality-assurance tests were performed according to the manufacturer's instructions. All scans were subsequently analyzed by a single trained investigator. The percentage of fat was calculated as fat mass $(\mathrm{kg})$ measured by DEXA divided by body weight (kg) measured by scale. The skeletal muscle compartments of the arms and legs were examined using the sub-region option of the software. The appendicular skeletal muscle mass was calculated as the sum of the arm and leg fat-free soft tissue. $^{16}$

\section{Aerobic exercise intervention}

The weekly volume of aerobic exercise consisted of 60-min exercise sessions of brisk walking twice per week (approximately 7-8 METs per week). During the 6-month intervention, a certified exercise trainer supervised the participants. Across the intervention period, the intensity was gradually increased and averaged $75 \pm 5 \%$ of the maximum heart frequency based on the Karvonen formula. ${ }^{17}$ While the participants walked, their heart rate (HR) was monitored and recorded using a HR monitor (Polar; Port Washington, NY, USA). At the conclusion of each exercise session, the subjects were asked to provide a rating of perceived exertion using the Borg scale. ${ }^{18}$ The subjects were asked to maintain a level of perceived exertion between 12 and 14 on the Borg scale (somewhat difficult), corresponding to a moderate level of intensity.

\section{Assessment of physical activity}

Typical physical activity was evaluated with the Physical Activity Scale for the Elderly (PASE). ${ }^{19}$ The total PASE score was computed by multiplying the amount of time spent in each activity (hours per day) or participation (yes/no) in an activity by the empirically derived item weights and summing over all activities. The item weights are based on a comparison with physical activity derived by regressing a component score developed from a 3-day physical activity diary and a global activity assessment.

\section{Statistical analyses}

The results are shown as the mean \pm s.d. The variables that were not normally distributed were log-transformed before analysis.

A repeated measures analysis of variance was performed to evaluate longitudinal changes in the main variables at baseline, 3 months and 6 months in the entire group, the normotensive group $(n=11)$ and the hypertensive $(n=10)$ group. To adjust PWVcf values for MAP, HR, TGs and systolic BP changes, the covariance analyses were conducted.

The unpaired $t$-test was used to compare variables between the subjects with and without hypertension at baseline.

We tested the study hypothesis considering only the physical activity effect between baseline PWVcf and 6-month PWVcf. A sample size of 10 subjects achieves $80 \%$ power to detect a mean paired difference of $2 \mathrm{~m} \mathrm{~s}^{-1}$ with an estimated s.d. of differences and a significance level (alpha) of 0.05 using a twosided, paired $t$-test. 
Table 1 Characteristics of the study population

\begin{tabular}{|c|c|c|c|c|}
\hline & $\begin{array}{l}\text { All }(\mathrm{n}=21) \\
\text { Mean } \pm \text { s.d. }\end{array}$ & $\begin{array}{c}\text { Normotensive }(\mathrm{n}=11) \\
\text { Mean } \pm \text { s.d. }\end{array}$ & $\begin{array}{l}\text { Hypertensive }(\mathrm{n}=10) \\
\text { Mean } \pm \text { s.d. }\end{array}$ & $\begin{array}{c}\text { P-value (normotensive vs. } \\
\text { hypertensive) }\end{array}$ \\
\hline Age (years) & $68.19 \pm 5.72$ & $67.0 \pm 6.14$ & $69.5 \pm 5.19$ & 0.33 \\
\hline Weight (kg) & $69.48 \pm 10.47$ & $70.91 \pm 13.05$ & $69.40 \pm 7.16$ & 0.97 \\
\hline BMI $\left(\mathrm{kg} \mathrm{m}^{-2}\right)$ & $28.63 \pm 4.69$ & $29.46 \pm 5.21$ & $28.20 \pm 3.10$ & 0.70 \\
\hline SAD $(\mathrm{cm})$ & $22.17 \pm 2.83$ & $22.06 \pm 3.64$ & $22.29 \pm 1.75$ & 0.86 \\
\hline Waist $(\mathrm{cm})$ & $89.33 \pm 10.46$ & $88.82 \pm 13.34$ & $89.90 \pm 6.71$ & 0.82 \\
\hline FM (kg) & $25.36 \pm 6.72$ & $24.91 \pm 8.10$ & $25.86 \pm 5.18$ & 0.76 \\
\hline FFM (kg) ${ }^{\mathrm{a}}$ & $41.18 \pm 4.48$ & $41.90 \pm 5.19$ & $40.38 \pm 36.4$ & 0.45 \\
\hline Total cholesterol (mg dl-1) & $227.76 \pm 38.69$ & $227.8 \pm 37.57$ & $226.3 \pm 43.48$ & 0.87 \\
\hline HDL cholesterol $\left(\mathrm{mgdl}^{-1}\right)$ & $53.67 \pm 11.47$ & $50.0 \pm 9.57$ & $57.6 \pm 12.95$ & 0.14 \\
\hline LDL cholesterol $\left(\mathrm{mgdl}^{-1}\right)$ & $145.48 \pm 33.33$ & $145.9 \pm 30.24$ & $141.9 \pm 37.80$ & 0.65 \\
\hline $\mathrm{TG}\left(\mathrm{mgdl} \mathrm{I}^{-1}\right)$ & $142.00 \pm 67.28$ & $159.3 \pm 80.93$ & $132.1 \pm 49.59$ & 0.53 \\
\hline Fasting glucose $\left(\mathrm{mgdl}^{-1}\right)$ & $87.59 \pm 9.40$ & $90.3 \pm 8.51$ & $84.0 \pm 9.52$ & 0.19 \\
\hline $\mathrm{HbA1}(\%)$ & $5.84 \pm 0.38$ & $5.93 \pm 0.41$ & $5.74 \pm 0.33$ & 0.27 \\
\hline HR (b.p.m.) & $79.36 \pm 9.22$ & $80.11 \pm 9.01$ & $78.30 \pm 4.07$ & 0.60 \\
\hline $\mathrm{SBP}(\mathrm{mm} \mathrm{Hg})$ & $145.95 \pm 23.93$ & $131.33 \pm 26.14$ & $156.11 \pm 22.55$ & 0.02 \\
\hline $\mathrm{DBP}(\mathrm{mm} \mathrm{Hg})$ & $82.43 \pm 10.12$ & $76.17 \pm 9.43$ & $85.10 \pm 9.94$ & 0.26 \\
\hline $\mathrm{PP}(\mathrm{mm} \mathrm{Hg})$ & $63.52 \pm 16.48$ & $54.45 \pm 13.4$ & $73.5 \pm 13.9$ & 0.05 \\
\hline $\mathrm{MAP}(\mathrm{mm} \mathrm{Hg})$ & $102.82 \pm 15.51$ & $98.15 \pm 12.4$ & $109.6 \pm 13.94$ & 0.06 \\
\hline $\operatorname{PWV} c r\left(\mathrm{~ms}^{-1}\right)$ & $9.19 \pm 1.14$ & $9.22 \pm 1.26$ & $9.14 \pm 1.00$ & 0.87 \\
\hline PWVcf $\left(\mathrm{ms}^{-1}\right)$ & $12.1 \pm 2.47$ & $11.74 \pm 2.38$ & $13.16 \pm 2.49$ & 0.05 \\
\hline
\end{tabular}

Abbreviations: BMI, body mass index; DBP, diastolic blood pressure; FFM, fat-free mass; FM, fat mass; HDL, high-density lipoprotein; HR, heart rate; LDL, low-density-lipoprotein; MAP, mean arterial pressure; PP, pulse pressure; PWVcf, pulse wave velocity carotid-femoral; PWVcr, pulse wave velocity carotid-radial; TG, triglycerides; SAD, sagittal abdominal diameter; SBP, systolic blood pressure.

aEvaluated by dual X-ray absorptiometry.

A significance level of $P<0.05$ was used throughout the study. All statistical analyses were performed using SPSS (version 16.0 for Windows). ${ }^{20}$

\section{RESULTS}

The main characteristics of the entire study sample and of the hypertensive and normotensive subjects are shown in Table 1.

Seventy-five percent of the sample was affected by mild hypercholesterolemia, $30 \%$ was affected by obesity and $45 \%$ was affected by hypertension. At baseline, no significant differences in the metabolic variables were observed between the hypertensive and normotensive subjects. As expected, the PWVcf and pulse pressure were significantly higher in the hypertensive subjects compared with the normotensive subjects $(P=0.02$ and 0.05 , respectively; Table 1$)$.

Table 2 shows the changes in the anthropometric, body composition and metabolic variables during the follow-up period for the entire group and for the normotensive and hypertensive subjects.

In the entire group $(n=21)$, a significant decline was observed in waist and SAD (both $P<0.05$ ) after 6 months, whereas fat-free mass, $\mathrm{BMI}$ and weight did not change significantly. The fat mass decreased after 3 months $(P<0.01)$, but this decline was not confirmed after 6 months.

After 6 months of the exercise program, triglycerides (TGs) $(P<0.01)$ decreased significantly, whereas there was no decrease in fasting blood glucose, total cholesterol or low-density-lipoprotein cholesterol. High-density lipoprotein cholesterol significantly increased at 3 months $(P<0.05)$, but this improvement was not confirmed at 6 months.

As shown in Table 3, a significant decline in the PWVcf $(P<0.05)$ but not in the PWCcr was observed throughout the study period. The PWVcf change remained significant even after adjusting for changes in MAP, HR TGs and waist circumference (Figure 1). Systolic and diastolic BP did not change during the follow-up period.
Considering the effect of physical activity separately in the hypertensive and normotensive subjects, we observed a significant decline in the PWVcf for hypertensive subjects and a nonsignificant tendency in normotensive subjects, with no change in BP.

The attendance at walking sessions decreased from 80.1 to $65.6 \%$ between the first ( $0-3$ months) and the second (3-6 months) periods of the study $(P<0.01)$, and there was no significant difference between the two groups for all the study variables. Finally, the PASE score did not change during the 6-month follow-up period for the hypertensive or normotensive subjects (data not shown in table).

\section{DISCUSSION}

The current data showed that a moderate 6-month aerobic exercise program reduced arterial stiffness independent of changes in TGs, MAP, HR and central adiposity.

A few studies have investigated the effect of physical activity on PWV in the elderly.

Observational studies have shown a linear association between physical activity and arterial compliance. Vaitkevicius et al. ${ }^{7}$ demonstrated a significant association, independent of age, between higher physical conditioning status and arterial stiffness, as assessed by aortic PWV and carotid AI, in 146 male and female volunteers, aged 21-96 years old. Furthermore, Aoyagi et al. ${ }^{21}$ found an association between lower arterial stiffness and habitual physical activity, independent of sex, age and mean arterial pressure, in a group of 89 men and 109 women (aged 65-84 years old).

An association between the time spent in light physical activity (1.1-2.9 METs daily) and arterial stiffening in a group of 109 unfit elderly subjects was shown in a recent cross-sectional study. ${ }^{11}$ Additionally, Madden et al. ${ }^{10}$ showed that aerobic exercise reduced both the PWVcf and PWVcr in a group of 36 elderly diabetic patients, whereas non-aerobic exercise did not demonstrate an effect. 


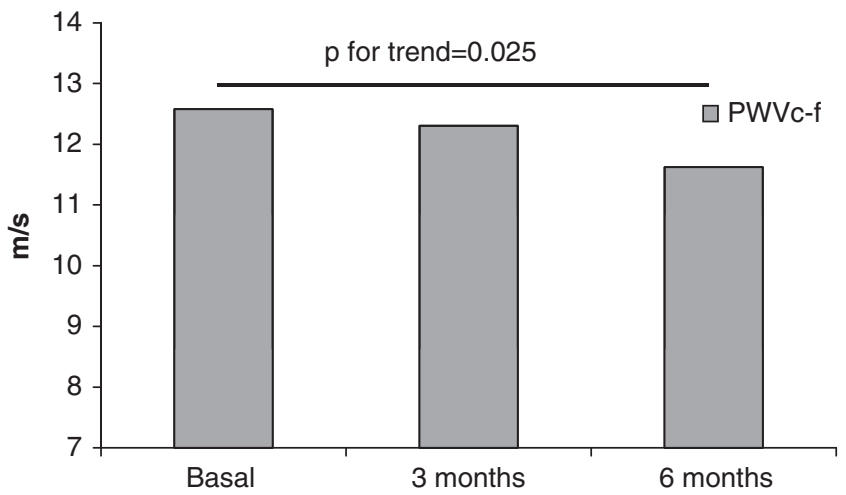

Figure 1 PWV carotid-femoral changes in the entire group $(n=21)$ during the 6-months follow-up period after adjustment for heart rate, mean arterial pressure, waist and triglycerides changes. A full color version of this figure is available at the Hypertension Research journal online.

The current study confirms and complements these results, as the significant PWVcf decline was independent of changes in TGs, MAP, HR and central adiposity and was observed after a 6-month period of a light amount of physical activity (that is, 60-min exercise sessions of brisk walking twice per week).

The present result that even a light amount of physical activity twice per week can ameliorate PWVcf is particularly relevant because the role of physical activity is strongly limited by the low rate of compliance, ${ }^{12}$ and it is possible to speculate that higher volumes of physical activity, in terms of intensity, duration and frequency per week, may lower compliance. Interestingly, the decrease in the PWVcf after 3 and 6 months of PA remained significant, even when the walking session attendance decreased from 80.1 to $65.6 \%$ in terms of duration and frequency per week, that is, with a physical activity intensity of roughly 4.5 METs.

As expected, the data show a beneficial effect of physical activity on the PWVcf but not on the PWVcr. These findings are in line with previous reports and confirm that the PWVcf, which explores elastic arteries such as the aorta, is more strictly related with $\mathrm{CV}$ risk than PWVcr. ${ }^{8,9}$

When the study population was subdivided into subjects with and without hypertension, the improvement in the PWVcf remained significant only in those subjects with hypertension; the normotensive subjects demonstrated a tendency toward improvement. This result is likely due to the higher values of $\mathrm{PWVcf}$ at baseline in the hypertensive compared with the normotensive subjects $\left(13.16 \pm 2.49 \mathrm{~m} \mathrm{~s}^{-1}\right.$ vs. $\left.11.67 \pm 2.50 \mathrm{~ms}^{-1}, P=0.05\right)$. Thus, the beneficial role of physical activity on the PWVcf in normotensive elderly individuals should be confirmed in a future study with a greater number of participants.

Several mechanisms can be advocated to support the current findings. Mechanical distension during aerobic exercise, through a 'stretching' of collagen fibers, may modify the cross-linking of these fibers. This process, in turn, increases the arterial compliance ${ }^{22}$ or modulation of the sympathetic tone of smooth muscle cells in the arterial wall. This effect is likely due to reduced vasoconstrictor tone or increased biodisponibility of $\mathrm{NO}^{8}$ which has been shown to regulate the arterial elasticity in human arteries. ${ }^{23}$

Some limitations of the present study should be noted. The study sample was small and limited to relatively healthy elderly women and thus cannot be considered representative of a normal aging population. As CV diseases incidence is higher in men than in women, the inclusion of men in the study population would have provided important additional information.

Second, we only evaluated surrogates of visceral fat, such as waist circumference and SAD. It is possible to hypothesize that a more precise measurement of fat distribution may have increased its role in arterial stiffness.

The subjects were not sedentary, as demonstrated by the mean PASE score at the beginning of the study $(146.72 \pm 24.11)$, which represented a moderate to high physical activity level.

Thus, the effect of the light physical activity program used in the current study protocol should be considered additive to the subjects' typical daily activity.

Finally, we did not evaluate the cardiorespiratory fitness of the subjects as $\mathrm{VO}_{2} \max$, for which the relation with arterial stiffness is well known. ${ }^{7}$

In conclusion, the present data showed that a moderate 6-month aerobic exercise program reduced arterial stiffness in a group of elderly women, and this result was independent of changes in TGs, systolic BP and central adiposity. These results suggest that even a moderate and well-accepted amount of physical activity is beneficial and should be considered for the prevention and reversal of subclinical vascular damage in the elderly, especially in hypertensive subjects.

\section{CONFLICT OF INTEREST}

The authors declare no conflict of interest.

1 Mora S, Cook N, Buring JE, Ridker PM, Lee IM. Physical activity and reduced risk of cardiovascular events: potential mediating mechanisms. Circulation 2007; 116 2110-2118.

2 Fernandez S, Scales KL, Pineiro JM, Schoenthaler AM, Ogedegbe G. A senior centerbased pilot trial of the effect of lifestyle intervention on blood pressure in minority elderly people with hypertension. J Am Geriatr Soc 2008; 56: 1860-1866.

3 Petrella RJ, Lattanzio CN, Demeray A, Varallo V, Blore R. Can adoption of regular exercise later in life prevent metabolic risk for cardiovascular disease? Diabetes Care 2005; 28: 694-701.

4 Blair SN, Kampert JB, Kohl HW, Barlow CE, Macera CA, Paffenbarger RS, Gibbons LW. Influences of cardiorespiratory fitness and other precursors on cardiovascular disease and all-cause mortality in men and women. JAMA 1996; 276: 205-210.

5 Wannamethee SG, Shaper AG. Walker M. Changes in physical activity, mortality, and incidence of coronary heart disease in older men. Lancet 1998; 351: 1603-1608.

6 Negri C, Bacchi E, Morgante S, Soave D, Marques A, Menghini E, Muggeo M, Bonora $E$, Moghetti $P$. Supervised walking groups to increase physical activity in type 2 diabetic patients. Diabetes Care 2010; 33: 2333-2335.

7 Vaitkevicius PV, Fleg JL, Engel JH, O'Connor FC, Wright JG, Lakatta LE, Yin FC, Lakatta EG. Effects of age and aerobic capacity on arterial stiffness in healthy adults. Circulation 1993; 88: 1456-1462.

8 Tanaka H, Safar ME. Influence of lifestyle modification on arterial stiffness and wave reflections. Am J Hyper tens 2005; 18: 137-144.

9 Casey DP, Pierce GL, Howe KS, Mering MC, Braith RW. Effect of resistance training on arterial wave reflection and brachial artery reactivity in normotensive postmenopausal women. Eur J Appl Physiol 2007; 100: 403-408.

10 Madden KM, Lockhart C, Cuff D, Potter TF, Meneilly GS. Short-term aerobic exercise reduces arterial stiffness in older adults with type 2 diabetes, hypertension, and hypercholesterolemia. Diabetes Care 2009; 32: 1531-1535.

11 Gando Y, Yamamoto K, Murakami H, Ohmori Y, Kawakami R, Sanada K, Higuchi M, Tabata I, Miyachi M. Longer time spent in light physical activity is associated with reduced arterial stiffness in older adults. Hypertension 2010; 56: 540-546.

12 Allender S, Hutchinson L, Foster C. Life-change events and participation in physical activity: a systematic review. Health Promot Int 2008; 23: 160-172.

13 Zamboni M, Turcato E, Armellini F, Kahn HS, Zivelonghi A, Santana H, BergamoAndreis IA, Bosello O. Sagittal abdominal diameter as a practical predictor of visceral fat. Int J Obes Relat Metab Disord 1998; 22: 655-660.

14 Laurent S, Kingwell B, Bank A, Weber M, Struijker-Boudier H. Clinical applications of arterial stiffness: therapeutics and pharmacology. Am J Hypertens 2002; 15: 453-458.

15 Visser M, Fuerst T, Lang T, Salamone L, Harris TB. Validity of fan-beam dual-energy $X$-ray absorptiometry for measuring fat-free mass and leg muscle mass. health, aging, and body composition study-dual-energy X-ray absorptiometry and body composition working group. J Appl Physiol 1999; 87: 1513-1520. 
16 Heymsfield SB, Smith R, Aulet M, Bensen B, Lichtman S, Wang J, Pierson Jr RN. Appendicular skeletal muscle mass: measurement by dual-photon absorptiometry. Am J Clin Nutr 1990; 52: 214-218.

17 Nelson ME, Rejeski WJ, Blair SN, Duncan PW, Judge JO, King AC, Macera CA, Castaneda-Sceppa C. Physical activity and public health in older adults: recommendation from the American College of Sports Medicine and the American Heart Association. Circulation 2007; 116: 1094-1105.

18 Borg G. Psychological bases of perceived exertion. Med Sci Sports Exerc 1982; 14: 377-381.

19 Washburn RA, Smith KW, Jette AM, Janney CA. The Physical Activity Scale for the Elderly (PASE): development and evaluation. J Clin Epidemiol 1993; 46: 153-162.
20 SPSS Inc. SPSS- $X$ User's Guide (2nd edn). McGraw-Hill, New York, 1986.

21 Aoyagi Y, Park H, Kakiyama T, Park S, Yoshiuchi K, Shephard RJ. Yearlong physical activity and regional stiffness of arteries in older adults: the Nakanojo Study. Eur J Appl Physiol 2010; 109: 455-464.

22 Seals DR, Desouza CA, Donato AJ, Tanaka H. Habitual exercise and arterial aging. J Appl Physiol 2008; 105: 1323-1332.

23 Kinlay S, Creager MA, Fukumoto M, Hikita H, Fang JC, Selwyn AP, Ganz P. Endothelium-derived nitric oxide regulates arterial elasticity in human arteries in vivo. Hypertension 2001; 38: 1049-1053. 